\title{
Stroke as the initial manifestation of Takayasu's arteritis: a Case report
}

\author{
Gupta AK ${ }^{1}$, Chaturvedi D², Mehrotra $\mathbf{Y}^{3}$, Trivedi HH $^{4}$ \\ ${ }^{1}$ Dr Anil Kumar Gupta, Director, Akshaya Heart Hospital, Bhopal, M.P .India, ${ }^{2}$ Dr Deepak Chaturvedi, Director, Akshaya \\ Heart Hospital, Bhopal, M.P .India, ${ }^{3}$ Dr Yogesh Mehrotra, Ex. Professor, Department of Medicine, Peoples College of \\ medical sciences and research Centre, Bhopal, MP, India. ${ }^{4}$ Dr Hari Har Trivedi, Ex. Professor, Department of Medicine, \\ Gandhi Medical College, Bhopal ,M.P., India
}

\begin{abstract}
Takayasu's arteritis is a chronic inflammatory and stenotic disease of medium and large sized arteries. We report clinical, laboratory and imaging findings of a 23 year old girl who presented with history of sudden onset of left hemiparesis, absent bilateral upper limb and right carotid pulsations, an elevated ESR of $58 \mathrm{~mm}$ at 1 hour,C-reactive protein level of 34.9 $\mathrm{mg} / \mathrm{L}$.Imaging studies showed non hemorrhagic acute infarct in territory of right MCA, concentric wall thickening and resultant stenosis of most of the branches of aortic arch. The diagnosis of Takayasu's arteritis was made and was put on steroids and anticoagulants. Patient showed a good clinical response.
\end{abstract}

Key Words: Takayasu's Arteritis, Stroke, Arteritis

\section{Introduction}

Takayasu's arteritis was first described by Dr. Mikito Takayasu (Japanese ophthalmologist) in 1905. It is a chronic inflammatory and stenotic disease of medium and large sized arteries of which the exact etiopathogenesis is unknown [ 1,2$]$. It is having strong predilection for the aortic arch and its branches. The involvement of the major branches of the aorta is much more marked at their origin than distally. It is pathologically characterized by granulomatous changes of the aorta and its major branches.

Takayasu's arteritis has been reported worldwide but more prevalent in Japan, Southeast Asia, India and Mexico. It is rare in US with an incidence of approximately 2.6 per million per year. It is more commonly seen in females in the second and third decade of life. Immunopathogenic mechanisms, the precise nature of which is uncertain, are suspected in this disease, while no certain cause is found in about one-third of young stroke victims [3]. It is also suggested that tuberculosis may have an association, in patients with Takayasu's arteritis [2]. Such patients may have either an active or a past tubercular infection.

The diagnosis should be suspected strongly in a young woman who develops a decrease or absence of peripheral pulses, discrepancies in blood pressure, and arterial bruits.

Manuscript received: $5^{\text {th }}$ July 2014

Reviewed: $12^{\text {th }}$ July 2014

Author Corrected: $24^{\text {th }}$ July 2014

Accepted for Publication: 26 $6^{\text {st }}$ July 2014
In 1990, the American College of Rheumatology proposed criteria for the diagnosis of Takayasu's arteritis. Six criteria were selected for the traditional format classification: onset at age less than or equal to 40 years, claudication of an extremity, decreased brachial artery pulse, greater than $10 \mathrm{~mm} \mathrm{Hg}$ difference in systolic blood pressure between arms, a bruit over the subclavian arteries or the aorta, and arteriographic evidence of narrowing or occlusion of the entire aorta, its primary branches, or large arteries in the proximal upper or lower extremities. The presence of 3 or more of these 6 criteria demonstrated a sensitivity of $90.5 \%$ and a specificity of $97.8 \%$ [4].

Though conventional angiography remained a diagnostic tool since earlier times but now Computed tomography angiography is found to be having a greater diagnostic accuracy than conventional angiography and it is having an advantage of being non -invasive technique as well as it is capable of demonstrating mural and luminal changes in both the aorta and aortic branches [5].

Takayasu arteritis can be divided into the following 6 types based on angiographic involvement [6].

- Type I - Branches of the aortic arch

- Type IIa - Ascending aorta, aortic arch, and its branches

- $\quad$ Type IIb - Type IIa region plus thoracic descending aorta 
- Type III - Thoracic descending aorta, abdominal aorta, renal arteries, or a combination

- Type IV - Abdominal aorta, renal arteries, or both

- $\quad$ Type V - Entire aorta and its branches

In Indian patients, there is more likelihood of involving the abdominal aorta including renal arteries and extending into the thoracic aorta.

Sometime neurologic involvement may be the initial presentation of the disease. The disease may have varied neurological manifestations. Patients may present with either headache or syncope or even blindness. Decreased blood supply to brain can give rise to varied neurological symptoms.

Approximately $10-20 \%$ of patients with Takayasu's arteritis are likely to have cerebrovascular accidents. Occlusion of the vertebral or carotid arteries may cause ischemic stroke. Patients with Takayasu disease may also develop intracranial aneurysms.

\section{Case Report}

A 23 year old left hander girl, presented with history of sudden onset of inability to move left upper limb and left lower limb, tilting of face to right side with slurring of speech in the early morning at about 5.30 AM. She correlated the complaints to exposure of cold as a result of sleeping on roof. She had previous history of headache off and on, mostly right side for 1 year for which she was treated as a case of Migraine.

On leading questionnaire she complaint of chest pain on walking which subsides on rest and take 1-1 $1 / 2$ hours to subside for last 3-4 months, and also, bilateral leg pains on walking for the same duration. Clinical examination at the time of admission showed absent bilateral upper limb pulses and absent right carotid pulsations, both lower limb pulses were normal. Lower limbs blood pressure was normal. There was no history suggestive of seizures.

The patient had history of operated for nasal polyps about 2 years back but the findings of that time did not mentioned the absence of pulses or any other significant findings. The patient had exanthema all over body, of about 2 months duration, the diagnosis of which was not clear.

There was a history of starting antitubercular to this patient for an eye nodule about 6 months back for which patient had taken incomplete course and stopped in 2 months only.
On examination, all the peripheral pulses of upper limbs were absent and in neck right carotid pulsations were

absent. There was no bruit. Pulses in lower limbs were palpable normally. Blood pressure in upper limbs was not recordable, Blood pressure measured in lower limbs, was 130/74 mm Hg.

Neurologic examination revealed left hemiparesis (power was grade-1 in left upper limb and grade- 2 in left lower limb), left sided hyperreflexia and extensor plantar response on left side. Optic fundus was normal. Cardiac examination was normal.

Hematological evaluation revealed hemoglobin of 9.2 gm $\%$, total leukocyte count $6500 / \mathrm{cmm}$ with $80 \%$ polymorphs and $15 \%$ lymphocytes, eosinophils $02 \%$. monocytes $03 \%$, Erythrocyte sedimentation rate was 58 $\mathrm{mm}$ at 1 hour.

C-reactive protein level was $34.9 \mathrm{mg} / \mathrm{L}$ (Normal upper limit $6 \mathrm{mg} / \mathrm{L}$ ), Serum ANA was negative. All other serum biochemical parameters were normal. Chest X-ray, electrocardiogram, echocardiogram and Doppler of heart were normal.

MRI brain with neck and brain angiography showed non hemorrhagic acute infarct in right lentiform nucleus and centrum semi ovale in the territory of right MCA.

MR neck angiography shows left CCA, ECA and ICA are normal in course and caliber with no evidence of stenosis seen, a short segment of concentric wall thickening of proximal part of left subclavian, left vertebral, right vertebral and right brachiocephalic trunk with wall thickening extending up to proximal right subclavian and CCA, resultant narrowing of the lumen showing no flow signals in the proximal segments. The right subclavian artery is diffusely attenuated (Fig $1 \& 3$ ).

MR brain angiography shows non visualization of right MCA. The right intracranial ICA and both ACA's are thin and communicating with left ICA.

Distal part of bilateral vertebral arteries, basilar artery and posterior cerebral arteries are also diffusely narrowed and communicating with left ICA by posterior communicating arteries. Overall most of the blood supply of brain is from left ICA (Fig $2 \& 3$ ).

CT angiography revealed diffuse concentric severe wall thickening of multiple major arch vessels causing severe 
stenosis/occlusion similar to MR angio as given above (Fig 4).

Aorta including ascending aorta, arch thoracic and abdominal aorta was normal in course, caliber, outline with normal origins of abdomen major arteries including bilateral renal arteries. Aortic bifurcation, Iliac, femoral arteries \& popliteal arteries were normal.

During her hospitalization the facial paresis worsened and also power in left lower limb decreased initially but improved to her baseline power, which was there at the time of hospitalization i.e. grade $2 / 5$ but left upper limb power worsened to grade $0 / 5$ at the time of discharge. Patient was discharged with rehabilitation exercises at

Brain angiography shows non-visualization of right MCA and attenuated right ICA

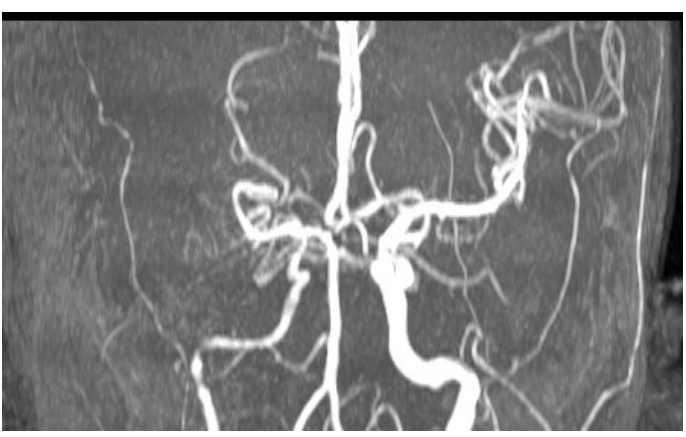

Figure 1: Angiography of Patient home under guidance of trained personnel. During her follow up after 1 week patient showed mild improvement in left lower limb. Subsequently after one

month of follow up after discharge patient had complete recovery of power in lower limb and also upper limb showed improvement with power grade $3 / 5$ at shoulder and elbow but power remained $0 / 5$ at wrist and no movements of fingers.

Latest follow up was done at 6 weeks interval which showed power grade $5 / 5$ in lower limb, grade $4 / 5$ in upper limb at all joints and good finger movements. Patient has shown a good response to steroid therapy and at present is on tapering doses of prednisolone.

MRI neck angiography shows attenuation of proximal aortic arch branches except left CCA. Retrograde filing of distal arterial segment from circle of Willis seen .

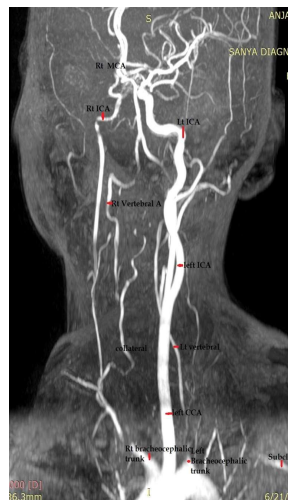

Figure 2: MR Angiography of patient

Neck angiography shows concentric wall thickening of proximal left vertebral and subclavian artery and brachiocephalic trunk extending to the proximal right CCA and right subclavian artery

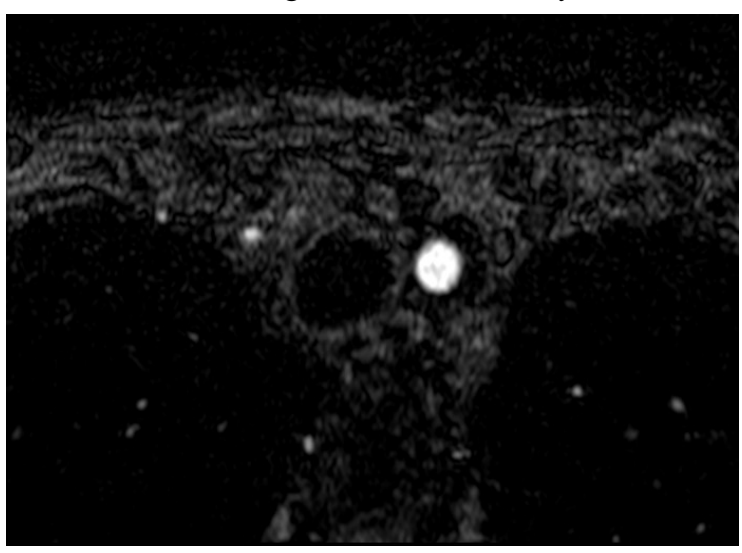

Figure 3: Neck Angiography 


\section{$\mathrm{CT}$ angiography of neck and brain shows normal caliber of left CCA with significant stenosis of proximal part of rest of the aortic arch branches. Both IJVs are normal.}
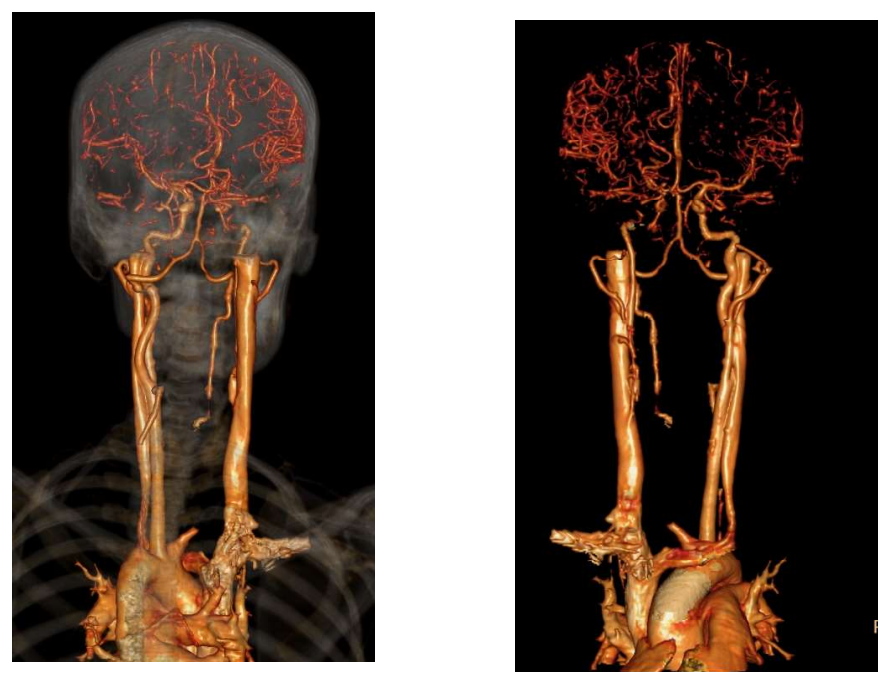

Figure 4: CT Angiography of Patient

\section{Discussion}

Our patient fulfilled American College of Rheumatology criteria for Takayasu's arteritis [4]. She had type-I (as per angiographic classification proposed by the International Cooperative Study on Takayasu's arteritis) disease showing severe involvement of aortic arch and its branches [6].

Our patient, at the time of presentation, had disabling left sided hemiplegia with slurred speech. Patient had concentric wall thickening of proximal part of right subclavian trunk with wall thickening extending upto right subclavian and common carotid artery. Right intracranial ICA was thin and there was non visualization of right MCA.

Diagnosis of Takayasu's arteritis was suspected as soon as patient was examined and found to be having absent upper limb pulses, absent right Carotid and soon after it, MRI with MR Angio was done the diagnosis was confirmed. Hence we again emphasize the importance of thorough clinical examination and examination of peripheral pulses in evaluating stroke in young.

Approximately $10-20 \%$ of patients with Takayasu's arteritis have ischemic stroke or transient ischemic attacks. Intracranial arterial vessels narrowing in Takayasu's arteritis could be due to either vasculitic involvement or a prior embolization into the vessel. The other postulated mechanisms may be occlusion of extracranial vessels, as a result of stenosis, hypertension and premature atherosclerosis.

Our patient had raised Erythrocyte sedimentation rate ie $58 \mathrm{~mm}$ at 1 hour. C-reactive protein level was $34.9 \mathrm{mg} / \mathrm{L}$ (Normal upper limit $6 \mathrm{mg} / \mathrm{L}$ ).

Although a physical examination alone is worthwhile to detect arterial disease but does not always localize or reveal the full extent of arteriographic lesions. Abnormal clinical finding pertaining to vascular system on physical examination are highly associated with the presence of arterial lesions, but a normal clinical examination do not exclude the possibility of arterial disease [7].

While various imaging techniques and blood levels of CRP and ESR remain the most useful tools and the gold standard in diagnosing and monitoring the progress of the disease, several biomarkers are found to be useful in recent studies, which include IL-6 [8], matrix metalloproteinase (MMP)-2, MMP-3, MMP-9 and pentraxin 3 (PTX3) [9].

But all of these biomarkers are still under investigation and none of them has showed a clear-cut correlation with the disease activity. Because of this reason, the Ishikawa clinical classification of Takayasu arteritis [10] is still widely used to determine prognosis and treatment plan. 
If the disease is diagnosed at an early stage, the disease could be controlled with the standard therapies as discussed earlier, and if critical vessels are involved the surgical intervention may be needed to prevent debilitating and irreversible complications such as massive cerebral ischemic infarction.

The patients of Takayasu's arteritis require long term immunosuppressive therapy to control vessel wall inflammation. The initial therapy consists of glucocorticoids, which can be used in combination with other immunosuppressive agents, such as azathioprine, methotrexate, or mycophenolate mofetil.

The goal of treatment is to induce and maintain disease remission. The remission is often defined as the absence of new arterial lesions, lack of systemic symptoms, and ability to taper prednisolone to less than $10 \mathrm{mg} / \mathrm{d}$ [11].

Our patient clinically responded very well to prednisolone therapy but it is a short time to assess. Patient needs certain more time to have a better assessment when she will undergo repeat investigations.

Certain patients, who do not respond to immunosuppressive therapy may require surgical intervention. Surgical treatment include endarterectomy, transluminal angioplasty and stenting and if needed bypass grafting.

Five-year survival is estimated to be $80 \%$ and largely depends on the clinical manifestations of disease and response to medical and surgical therapy [12].

\section{Conclusion}

Takayasu's Arteritis should be suspected in all young patients who present with stroke, especially in young females and that too presenting with differential blood pressure between arms or absent pulses, a bruit over the subclavian arteries or the aorta, as this disease is fairly responsive to medical management.

It also emphasizes the importance of thorough clinical examination as this will pick up disease at an early stage, which is necessary to prevent any further systemic complications, which may be devastating.

Funding: Nil

Conflict of interest: None

Permission from IRB: Yes

\section{References}

1. Kerr GS, Hallahan CW, Giordano J, Leavitt RY, Fauci AS, Rottem M, Hoffman GS. Takayasu arteritis.Ann Intern Med. 1994 Jun 1;120(11):919-29.

2. Johnston SL, Lock RJ, Gompels MM. Takayasu arteritis: a review. J Clin Pathol. 2002 Jul;55(7):481-6.

3. Hart RG, Miller VT. Cerebral infarction in young adults: a practical approach.Stroke. 1983 JanFeb;14(1):110-4.

4. Arend WP, Michel BA, Bloch DA, Hunder GG, Calabrese LH, Edworthy SM, Fauci AS, Leavitt RY, Lie JT, Lightfoot RW Jr, The American College of Rheumatology 1990 criteria $f$ or the classification of Takayasu arteritis. Arthritis Rheum. 1990 Aug;33(8):1129-34.

5. Khandelwal N, Kalra N, Garg MK, Kang M, Lal A, Jain S, Suri S. Multidetector CT angiography in Takayasu arteritis. Eur J Radiol. 2011 Feb;77(2):369-74. doi: 10.1016/j.ejrad.2009.08.001. Epub 2009 Aug 31.

6. Hata A, Noda M, Moriwaki R, Numano F. Angiographic findings of Takayasu arteritis: new classification. Int J Cardiol. Aug 1996;54 Suppl:S155-63.

7. Grayson PC, Tomasson G, Cuthbertson D, Carette S, Hoffman GS, Khalidi NA, Langford CA, McAlear CA, Monach PA, Seo P,Warrington KJ, Ytterberg SR, Merkel PA. Vasculitis Clinical Research Consortium Association of vascular physical examination findings and arteriographic lesions in large vessel vasculitis. J Rheumatol. 2012 Feb;39(2):303-9. doi: 10.3899/jrheum.110652. Epub 2011 Dec 15.

8. Villa I, Agudo Bilbao M, Martínez-Taboada VM. Advances in the diagnosis of large vessel vasculitis: identification of biomarkers and imaging studies. Reumatol Clin. 2011 Dec;7 Suppl 3:S22-7. doi: 10.1016/j.reuma.2011.09.003. Epub 2011 Nov 25.

9.Ishihara T, Haraguchi G, Tezuka D, Kamiishi T, Inagaki H, Isobe M. Diagnosis and assessment of Takayasu arteritis by multiple biomarkers. Circ J. 2013;77(2):477-83. Epub 2012 Oct 26.

10.Ishikawa K. Natural history and classification of occlusive thromboaortopathy (Takayasu's disease). Circulation. 1978 Jan;57(1):27-35. 
11. Koening CL, Langford CA. Takayasu's arteritis. Curr Treat Options Cardiovasc Med. 2008 Apr;10(2):164-72.
12.Subramanyan R, Joy J, Balakrishnan KG. Natural history of aortoarteritis (Takayasu's disease). Circulation. 1989 Sep;80(3):429-37.

How to cite this article?

Gupta AK, Chaturvedi D, Mehrotra Y, Trivedi HH. Stroke as the initial manifestation of Takayasu's arteritis: a Case report. Int J Med Res Rev 2014;2(4):397-402. doi:10.17511/ijmrr.2014.i04.24 